\title{
DEVELOPMENT OF THE ULTRA-LIGHT STRETCHED LENS ARRAY
}

\author{
M.J. O'Neill ${ }^{1}$, A.J. McDanal ${ }^{1}$, P.J. George ${ }^{2}$, M.F. Piszczor ${ }^{2}$, D.L. Edwards ${ }^{3}$ \\ D.T. Hoppe ${ }^{3}$, M.I. Eskenazi ${ }^{4}$, M.M. Botke ${ }^{4}$, P.A. Jaster ${ }^{5}$, H.W. Brandhorst ${ }^{6}$ \\ ${ }^{1}$ ENTECH, Inc., 1077 Chisolm Trail, Keller, TX 76248, ${ }^{2}$ NASA GRC, 21000 Brookpark Rd., Cleveland, OH 44135, \\ ${ }^{3}$ NASA MSFC, ED31, Huntsville, AL 35812, ${ }^{4}$ ABLE Engineering, 7200 Hollister Ave., Goleta, CA 93117, \\ ${ }^{5} 3 \mathrm{M}$ Center, 236-1B-38, St. Paul, MN 55144, ${ }^{6}$ Space Research Institute, 231 Leach Center, Auburn Univ., AL 36849
}

\begin{abstract}
At the last IEEE-PVSC, the new stretched lens array (SLA) concept was introduced. Since that conference, the SLA team has made significant advances in the SLA technology, including component-level improvements, array-level optimization, space environment exposure testing, and prototype hardware fabrication and evaluation. This paper will describe the evolved version of the SLA, highlighting the improvements in the lens, solar cell, rigid panel structure, and complete solar array wing. The near-term SLA will provide outstanding wing-level performance: $>180 \mathrm{~W} / \mathrm{kg}$ specific power, >300 W/sq.m. power density, $>300 \mathrm{~V}$ operational voltage, and excellent durability in the space environment.
\end{abstract}

\section{INTRODUCTION AND BACKGROUND}

Since 1986, ENTECH and NASA have been developing and refining space photovoltaic arrays using refractive concentrator technology [1]. Unlike reflective concentrators, these refractive Fresnel lens concentrators can be configured to minimize the effects of shape errors, enabling straightforward manufacture, assembly, and operation on orbit. By using a unique arch shape, these Fresnel lenses provide more than 100X larger slope error tolerance than either reflective concentrators or conventional flat Fresnel lens concentrators [2].

In the early 1990's, the first refractive concentrator array was developed and flown on the PASP+ mission, which included a number of small advanced arrays (3). The refractive concentrator array used ENTECH mini-dome lenses over Boeing mechanically stacked multi-junction (MJ) cells ( $\mathrm{GaAs}$ over $\mathrm{GaSb}$ ). The mini-dome lenses were made by ENTECH from space-qualified silicone (DC 93500 ), and coated by Boeing and OCLI to provide protection against space ultraviolet (UV) radiation and atomic oxygen (AO). This array performed extremely well throughout the year-long mission in a high-radiation geostationary transfer orbit (GTO), validating both the high performance and radiation hardness of the refractive concentrator approach (3). In addition, in high-voltage space plasma interaction experiments, the refractive concentrator array was able to withstand cell voltage excursions to $500 \mathrm{~V}$ relative to the plasma with minimal environmental interaction [3].
In the middle 1990's, ENTECH and NASA developed a new line-focus Fresnel lens concentrator, which is easier to make and more cost-effective than the mini-dome lens concentrator. Using a continuous roll-to-roll process, 3M can now rapidly mass-produce the line-focus silicone lens material in any desired quantity.

In 1994, AEC-ABLE joined the refractive concentrator team and led the development of the SCARLET® (Solar Concentrator Array using Refractive Linear Element Technology) solar array [4]. SCARLET uses a small (8.5 $\mathrm{cm}$ wide aperture) silicone Fresnel lens to focus sunlight at 8X concentration onto radiatively cooled triple-junction cells. Launched in October 1998, a $2.5 \mathrm{~kW}$ SCARLET array powered both the spacecraft and the ion engine on the NASA/JPL Deep Space One probe, shown in Fig. 1. SCARLET achieved over $200 \mathrm{~W} / \mathrm{sq} . \mathrm{m}$. areal power density and over $45 \mathrm{~W} / \mathrm{kg}$ specific power [5]. With SCARLET working flawlessly, Deep Space 1 had a spectacularly successful rendezvous with the comet, Borrelly, in September 2001, capturing the highest-resolution images of a comet to date and other unprecedented comet data.

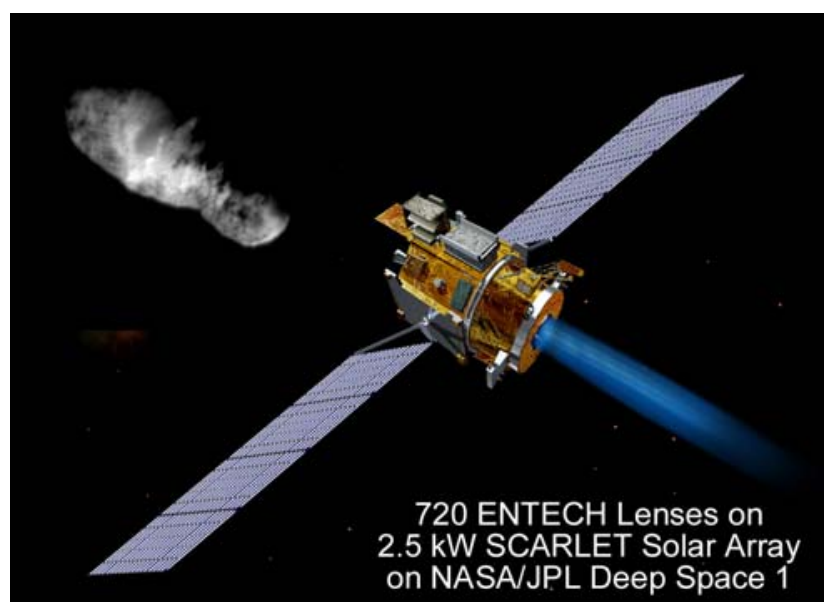

Fig. 1 - SCARLET Array on NASA/JPL Deep Space 1

Over the past three years, the team, including Auburn's Space Research Institute, has been developing a new space concentrator array technology, called the stretched lens array (SLA). SLA provides even higher performance than SCARLET at dramatically reduced mass and cost [6]. The following paragraphs describe SLA progress in the past 20 months since the last IEEE PVSC paper [6]. 


\section{THE STRETCHED LENS}

The patented SLA uses a thin, flexible, linear Fresnel lens optical concentrator to focus color-mixed sunlight onto multi-junction solar cells [ 7 and 8 ]. The lens is made by $3 \mathrm{M}$ using a high-speed, continuous, roll-to-roll process, from space-qualified silicone rubber material (DC 93-500). For the SCARLET array and for the original SLA, the lens material was about 275 microns thick, comprising 100 micron tall prisms on a 175 micron base. In 2001, 3M conducted a trial run of much thinner lens material, comprising the same 100 micron tall prisms on an 87 micron base. Since the prism layer is half void, the total mass of the thinner lens material is $40 \%$ less than for the thicker material. Despite this drastic reduction in thickness and mass, the new ultra-thin lens material maintained the same high optical efficiency of $92 \%+1 \%$, when tested at 9X geometric concentration using a GalnP single-junction reference cell (Fig. 2).

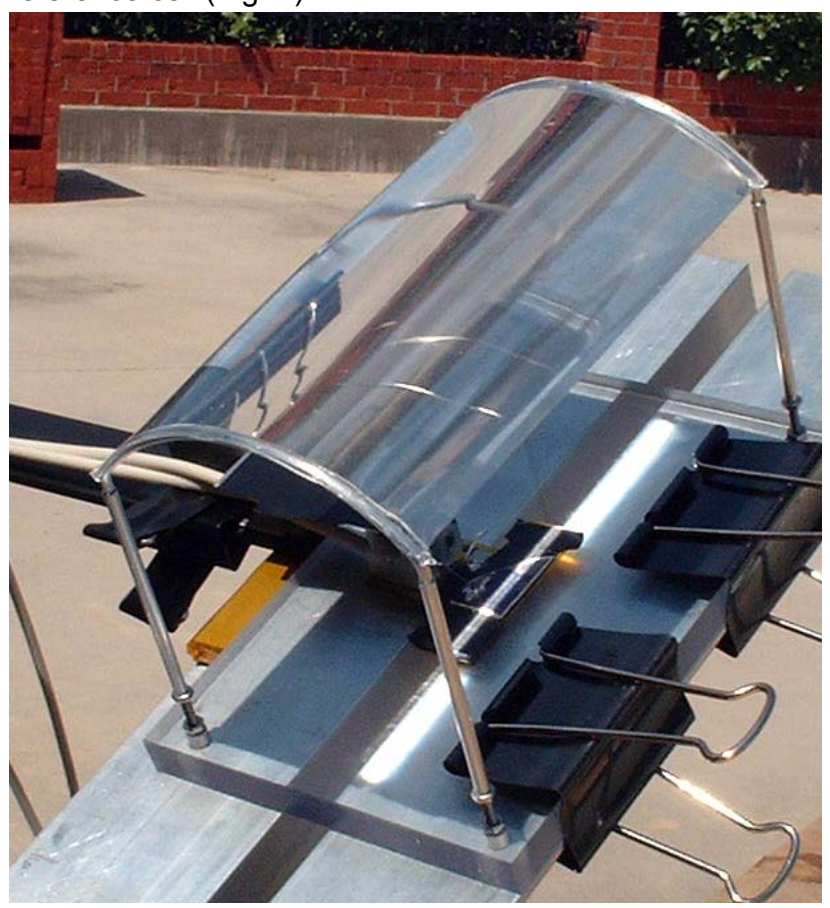

Fig. 2 - Ultra-Thin Lensfilm Test

ABLE thermal-cycled a deployed thin lens sample with no mechanical failure, and NASA Marshall exposed a deployed thick lens sample to electrons with no measurable optical loss, as summarized below:

\begin{tabular}{|c|c|c|}
\hline Type of Test & Exposure & Rationale \\
\hline $\begin{array}{c}\text { Thermal Cycling } \\
\text { (Thin Lensfilm) }\end{array}$ & $\begin{array}{c}1,800 \text { Cycles from } \\
-180 \text { to }+120 \mathrm{C}\end{array}$ & $\begin{array}{c}\text { Equivalent to } 20 \text { Years on } \\
\text { GEO }\end{array}$ \\
\hline $\begin{array}{c}\text { Electrons } \\
\text { (Thick Lensfilm) }\end{array}$ & $1.0 \times 10^{15} @ 1 \mathrm{MeV}$ & $\begin{array}{c}\text { Standard Solar Cell Test } \\
\text { (Additional Testing Planned) }\end{array}$ \\
\hline
\end{tabular}

Plano-plano samples of the silicone material used to make the lens have also been subjected to combined vacuum ultraviolet (VUV, from 119 to 200 micron wavelength) and near ultraviolet (NUV, from 200 to $400 \mathrm{~nm}$ wavelength) exposure testing by NASA Marshall. One recent lens material sample was coated by ZC\&R Optical Coatings with a multi-layer UV-rejection (UVR) coating like the coating successfully used on the silicone mini-dome lenses on the PASP+ flight test, with very encouraging results (Fig. 3). Despite the very low wavelength output of the VUV lamp, with $80 \%$ of the lamp power below $170 \mathrm{~nm}$ wavelength, the transmittance loss was relatively small after nearly 7,800 equivalent sun hours $(E S H)$ of combined NUV/VUV exposure. Indeed, when the spectral transmittance curve is convolved with the quantum efficiency curve of the top cell (GalnP) in the triple-junction stack, the net current loss under AMO sunlight is about $5 \%$. With proper tailoring of the initial top-to-middle cell current ratio, the effective loss in total cell power output can be less than $2.5 \%$ due to this effect. Additional work is ongoing to better understand the UV degradation phenomena and to thereby improve the UVR coating. In addition, a flight test of coated versus uncoated lens material is badly needed to quantify the actual UV degradation effect in space, since this effect is very difficult to accurately simulate in ground testing.

UV-Rejection-Coated DC 93-500 Spectral Transmittance

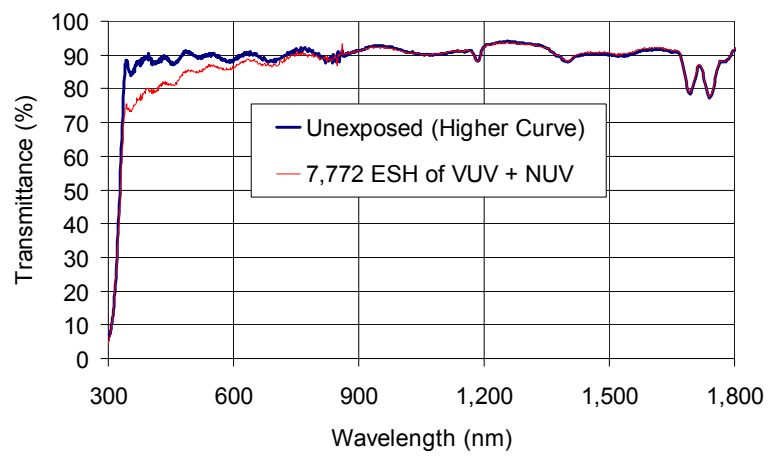

Fig. 3 - Combined VUV/NUV Exposure Results

\section{THE SOLAR CELLS}

Both Spectrolab and EMCORE have recently provided improved triple-junction (GalnP/GaAs/Ge) concentrator cells for the SLA. The Spectrolab cells are fully described in another paper at this conference [9]. The concentrator cells have a $1.0 \mathrm{~cm}$ active width between parallel busbars, corresponding to $8.5 \mathrm{X}$ geometric concentration ratio, since the lens has an aperture width of $8.5 \mathrm{~cm}$. The $3.5-\mathrm{cm}$-long Spectrolab cells included 94 identical cells with parallel gridlines spaced on 254 micron centers, to be compatible with one version of ENTECH's prismatic cell cover, which eliminates the normal gridline shadowing loss. Spectrolab tested these 94 bare cells at both 1 AM0 sun irradiance and 8 AMO suns irradiance, with the results shown in Fig. 4. As expected, the cell efficiency is significantly higher at 8 suns irradiance. The average cell performance gain due to concentration is $11 \%$ for all 94 cells. Interestingly, this gain effectively offsets the transmittance loss of the lens, boosting SLA's outstanding performance. 


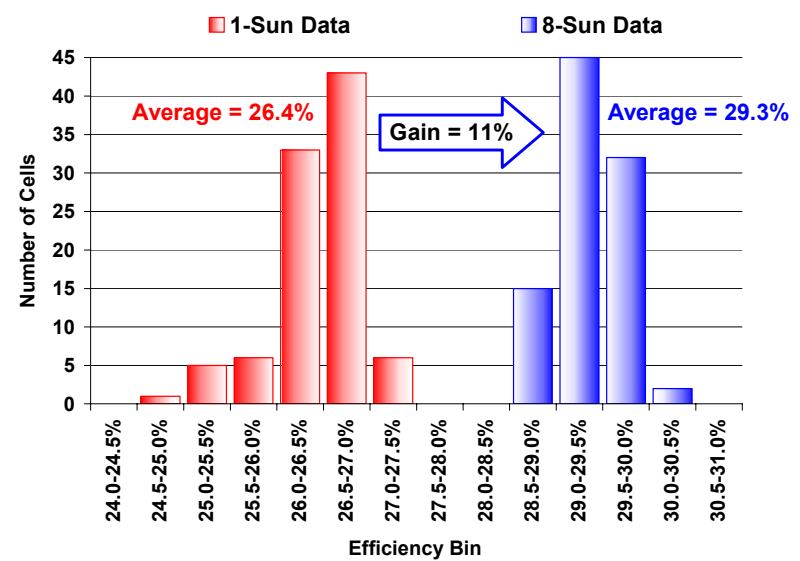

Fig. 4 - Spectrolab Bare Cell Performance

Note that the $11 \%$ relative gain in cell efficiency at 8 suns compared to 1 sun is accompanied by a tightening of the cell performance distribution. Only 4 cell efficiency bins are required to contain all 94 cells at 8 suns, compared to 6 cell efficiency bins at 1 sun. The $29.3 \%$ average bare cell efficiency is expected to increase to about $31 \%$ when prismatic cell covers are applied to these cells, considering the $6 \%$ grid shadowing factor. As discussed in the following section, Spectrolab cells equipped with prismatic covers have demonstrated outstanding performance in recent NASA Glenn testing.

The 4.0-cm-long EMCORE sample cells use a closer grid spacing (127 microns on centers), with a correspondingly higher grid shadowing loss (about 16\%). Prismatic cell covers are essential to good performance for these highly metallized cells. As discussed in the following section, EMCORE cells equipped with the prismatic covers have also provided outstanding performance in recent NASA Glenn testing.

\section{THE COMBINED LENS/CELL MODULES}

In recent months, several combined lens/cell modules have been fabricated and flown on the NASA Glenn Lear Jet to determine their AMO short-circuit currents. These currents have then been used to calibrate NASA Glenn's large area pulsed solar simulator (LAPSS) during full IV curve testing of the same SLA modules. SLA modules using the new ultra-thin lensfilm and prism-covered cells from both Spectrolab and EMCORE have been tested by NASA Glenn using this combined Lear/LAPSS approach, with outstanding results. Fig. 5 shows one of the SLA modules during outdoor ground testing at ENTECH, prior to delivery to NASA Glenn for Lear and LAPSS testing. The unit was sized to fit within the sun-pointing tube on the NASA Glenn Lear Jet. Fig. 6 shows the NASA-Glennmeasured AMO IV curve for the SLA module using a prism-covered EMCORE cell. Fig. 7 shows the NASAGlenn-measured AMO IV curve for the SLA module using a prism-covered Spectrolab cell. Note that both modules have a net electrical conversion efficiency over $27 \%$ under AM0 sunlight at room temperature (25C). Since these SLA module efficiency values are based on the measured

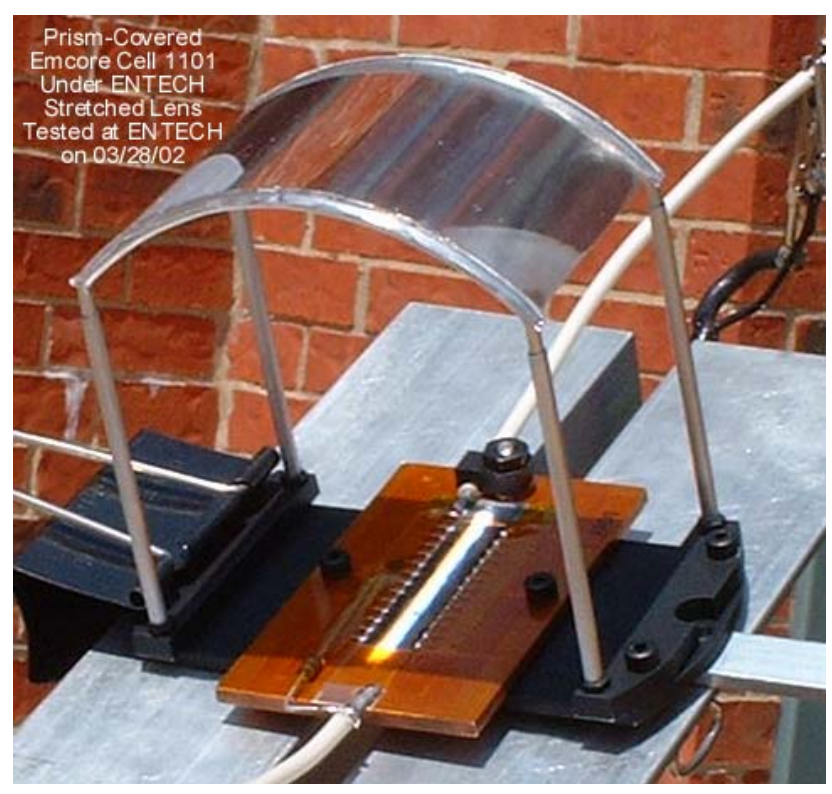

Fig. 5 - SLA Module for Lear/LAPSS Testing

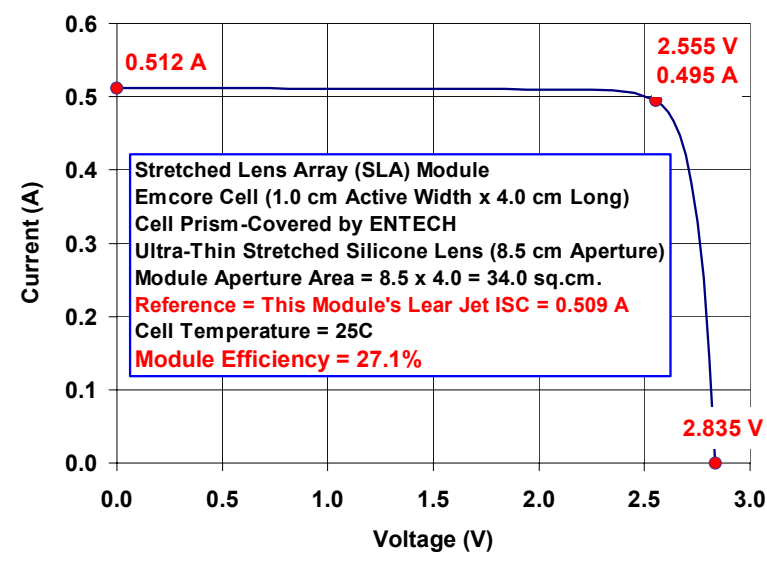

Fig. 6 - 27\% SLA Module Using EMCORE Cell

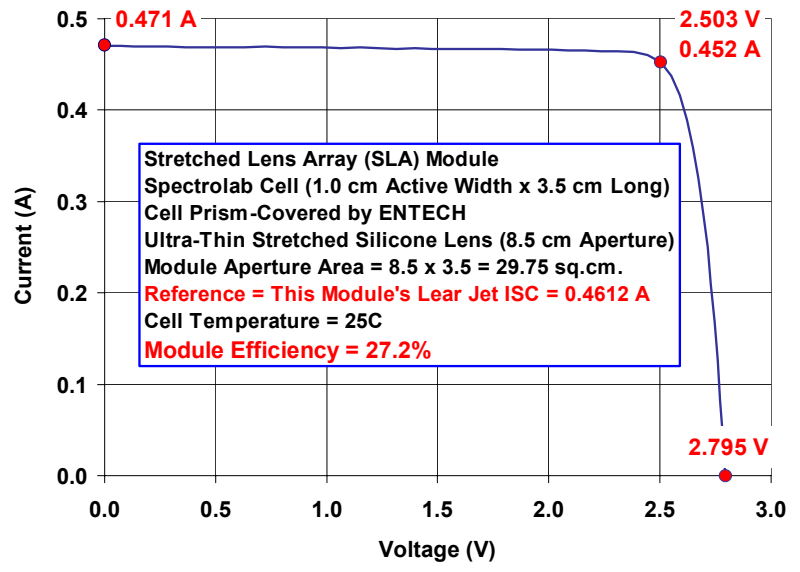

Fig. 7 - 27\% SLA Module Using Spectrolab Cell 
short-circuit currents from the Lear Jet flights, they also include any optical losses due to imperfect sun-pointing during the Lear Jet flight. Also, Auburn has conducted micrometeoroid impact tests on other lens/cell hardware, with the cells biased to $1,000 \mathrm{~V}$ relative to simulated space plasma, without electrical discharge problems.

\section{THE SOLAR ARRAY WING}

Initially, the SLA team was considering a flexible blanket solar array wing approach for SLA [6]. This approach has now been superseded by a more conventional rigid panel wing approach, with spring-driven, self-deploying end arches to support the stretched lenses (Fig. 8). By using ultra-light, single-face-sheet, picture-frame panels for all but the innermost and outermost panels of the wing, the rigid panel wing is superior to the blanket wing in performance, mass, cost, flight heritage, and marketability. A subscale prototype four-panel wing has been fabricated and evaluated mechanically, as shown at the bottom of Fig. 8. This wing will be equipped with a full complement of lenses and several "live" solar cell strings later this year.

\section{WING-LEVEL PERFORMANCE}

A detailed analysis has been conducted for a $7 \mathrm{~kW}$ rigidpanel SLA wing, resulting in the following full-wing performance estimates (on-orbit beginning of life, BOL):

\begin{tabular}{|l|c|}
\hline Areal Power Density & 300 W/sq.m. \\
\hline Specific Power & 180 W/kg \\
\hline Stowed Power Density & $9 \mathrm{~kW} / \mathrm{cu} . \mathrm{m}$. \\
\hline Stiffness (Stowed/Deployed) & $40 \mathrm{~Hz} / 0.1 \mathrm{~Hz}$ \\
\hline Operational Voltage & $300 \mathrm{~V}$ \\
\hline
\end{tabular}

\section{ACKNOWLEDGEMENT}

The SLA development reported in this paper has been supported by several NASA contracts.

\section{REFERENCES}

[1] M.F. Piszczor and M.J. O'Neill, "Development of a Dome Fresnel Lens/GaAs Photovoltaic Concentrator for Space Applications," 19th IEEE-PVSC, 1987.

[2] M.J. O'Neill, "Silicon Low-Concentration, Line-Focus, Terrestrial Modules," Chapter 10 in Solar Cells and Their Applications, John Wiley \& Sons, 1995.

[3] H. Curtis and D. Marvin, "Final Results from the PASP Plus Flight Experiment," 25th IEEE-PVSC, 1996.

[4] P.A. Jones et al., "The SCARLET Light Concentrating Solar Array," 25th IEEE-PVSC, 1996.

[5] D.M. Murphy, "The SCARLET Solar Array: Technology Validation and Flight Results," Deep Space 1 Technology

Validation Symposium, Pasadena, 2000.

[6] M.J. O'Neill et al., "The Stretched Lens Ultralight Concentrator Array," 28th IEEE-PVSC, 2000.

[7] M.J. O'Neill, "Stretched Fresnel Lens Solar Concentrator for Space Power," U.S. Patent 6,075,200, 2000.

[8] M.J. O'Neill, "Color-Mixing Lens for Solar Concentrator System and Methods of Manufacture and Operation Thereof," U.S. Patent 6,031,179, 2000.

[9] A. Stavrides et al., "Fabrication of High-Efficiency III-V MultiJunction Solar Cells for Space Concentrators," 29th IEEE-PVSC, 2002.
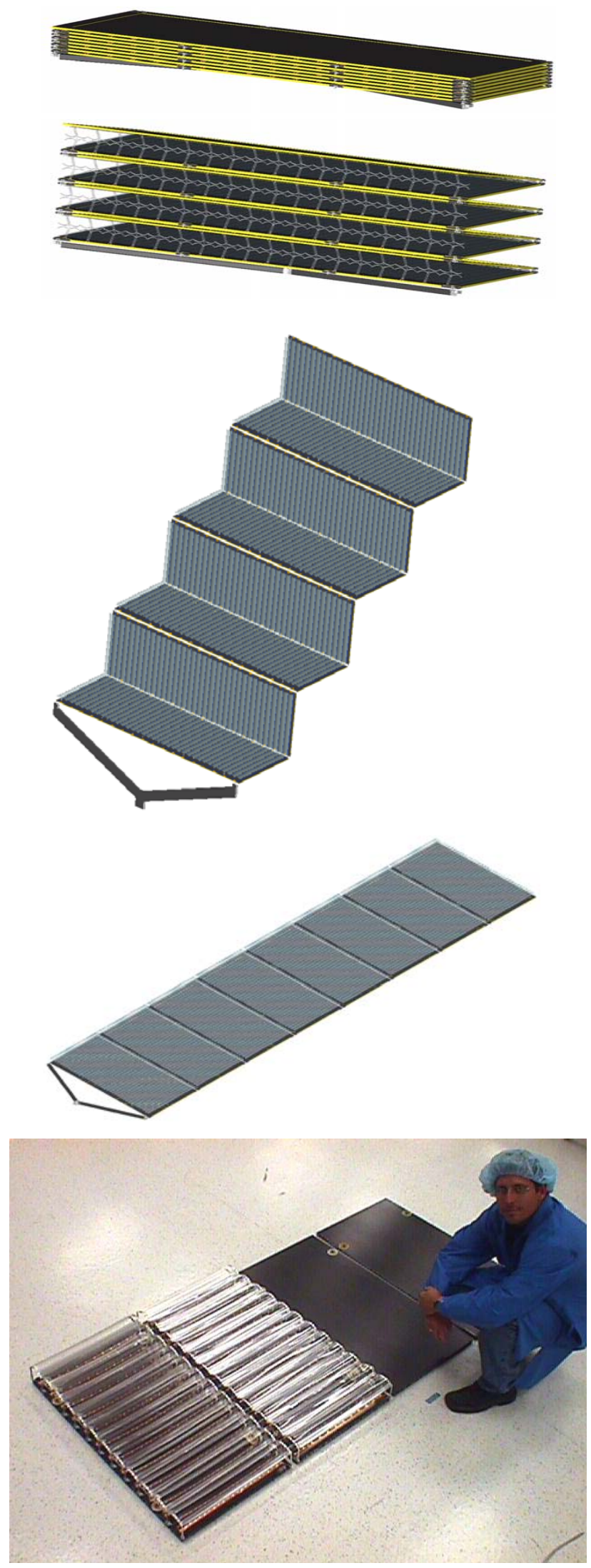

Fig. 8 - Rigid Panel SLA Wing Approach 